\title{
The paediatric rheumatologist and orphan disease - a story without happy ending
}

\author{
Justyna Roszkiewicz, Małgorzata Biernacka-Zielińska, Elżbieta Smolewska \\ Department of Paediatric Rheumatology, Medical University of Lodz, Poland
}

\begin{abstract}
Orphan diseases are not a common challenge in the everyday practice of the rheumatologist. Despite their extremely rare occurrence one of the patients under our care developed one of them - neuronal ceroid lipofuscinosis, the most frequent neurodegenerative disease observed in the paediatric population. We report a case of 2-year-old girl diagnosed with oligoarticular form of juvenile idiopathic arthritis treated in our Department with steroids and methotrexate and staying in the stage of disease remission. During routine checkups at Outpatient Clinic we observed progressive deterioration of girls neurological condition resulting in ataxia, gait disturbances with no rheumatological cause behind and speech impairment. The appearance of the symptoms was accompanied by frequent episodes of epileptic seizures, with little clinical improvement on combined antiepileptic treatment. Magnetic resonance imaging that we performed showed a picture highly suggestive of neuronal ceroid lipofuscinosis - atrophy of the patients cerebrum and cerebellum. Genetic testing conducted resulted in the diagnosis of late infantile neuronal ceroid lipofuscinosis (LINCL).
\end{abstract}

Key words: juvenile idiopathic arthritis, neuronal ceroid lipofuscinosis, CLN2, orphan diseases.

\section{Introduction}

Orphan diseases, defined by the European Commission on Public Health as "life-threatening or chronically debilitating diseases occurring with the prevalence lower than 1 in 2,000 people" are a challenge for every clinician. It is needless to mention how crucial it is to be aware of their clinical picture and distinguish whether the symptoms presented by our patient are inscribed in the natural course of their rheumatic disease or they may be the first indicators of underlying disease of a different aetiology. We herein describe a case of a girl diagnosed with juvenile idiopathic arthritis (JIA) who during routine checkups at Paediatric Rheumatology Outpatient Clinic presented with features of progressive neurodegeneration and was consequently diagnosed with a lysosomal storage disease - late infantile neuronal ceroid lipofuscinosis (LINCL/CLN2). Up to our knowledge, this is the first case of JIA and LINCL coexistence in one patient.

\section{Case study}

A previously healthy 2-year-old girl was admitted to the Department of Paediatric Rheumatology with bilateral inflammation of ankle joints. According to her medical history, appearance of this symptom was preceded by respiratory tract infection treated symptomatically by the GP. Basic laboratory tests performed at that moment revealed elevated values of inflammatory markers (leukocytes - 13 000, CRP - $10.6 \mathrm{mg} / \mathrm{dl}$, ESR - $45 \mathrm{~mm} / \mathrm{h}$ ) and presence of IgM antibodies against Chlamydophila and Mycoplasma pneumoniae. Antinuclear antibodies and rheumatoid factor were absent. Antibiotic and non-steroidal anti-inflammatory drug (NSAID) treatment was introduced with clinical improvement.

After two months, the girl was re-admitted to the hospital with the recurrence of those symptoms. Her ankle joints were swollen again, and swelling of inter phalangeal joints of her $3^{\text {rd }}$ finger of the right hand also appeared. In the laboratory tests inflammatory marker 
Table I. Laboratory test findings at the moment of JIA diagnosis

\begin{tabular}{|cc|}
\hline \multicolumn{2}{|c|}{ Laboratory tests results } \\
\hline ESR & $40 \mathrm{~mm} / \mathrm{h}(\mathrm{N} \mathrm{0}-12)$ \\
\hline CRP & $9.9 \mathrm{mg} / \mathrm{dl}(\mathrm{N} \mathrm{0-5)}$ \\
\hline ANA & $1: 160$ \\
\hline RF & negative \\
\hline Anti-CCP & negative \\
\hline
\end{tabular}

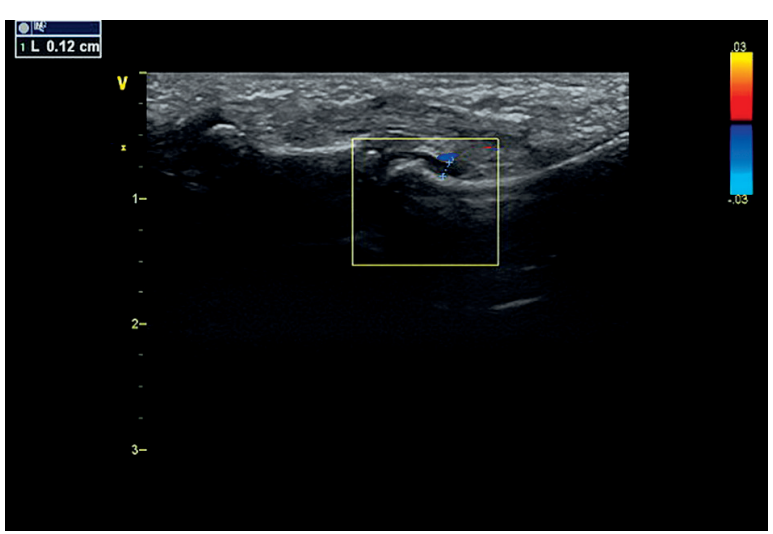

Fig. 1. Ultrasound picture of patients ankle joint.

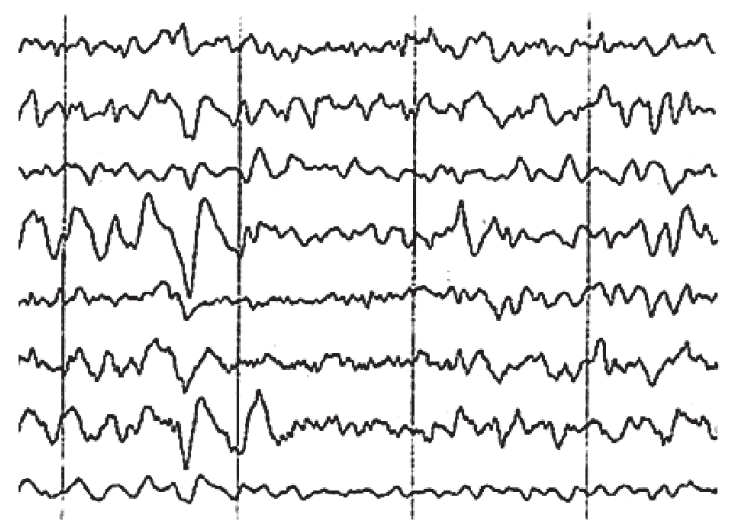

Fig. 2. EEG with registered seizure activity.

values were outside the normal ranges as shown in Table I. Ultrasonography of the peripheral joints was also performed, it has revealed significant thickening of the synovial membrane and features of exudation to the synovial cavity within all the joints affected by the inflammatory process (Fig. 1). Due to the overall clinical picture and laboratory tests findings the girl was diagnosed with JIA and treatment with systemic steroids (5 mg of prednisone daily) and disease-modifying anti-rheumatic drug (DMARD) (5 mg of methotrexate weekly) was introduced.
The treatment was successful, and for the year following the diagnosis JIA remained in remission. The patient had no signs of peripheral joints' involvement in the inflammatory process and laboratory test findings were within the normal range, which has led to a decrease of her daily steroid intake down to $2.5 \mathrm{mg}$ of prednisone daily.

The worst was yet to come, though - three months later our patient was admitted to the Emergency Department after an incident of epileptic seizure and accompanying loss of consciousness. The occurrence of the seizure was first thought to be the adverse effect of methotrexate treatment, which led to the drug's suspension. Despite this fact, another episode of seizures appeared during the routine hospitalization in our Department - the seizures were focal and asymmetrical, lasted for about 1 minute. Laboratory tests conducted immediately after the seizure showed no signs of electrolyte imbalance, and the glucose value was also within the normal range. The patient was consulted by a neurologist and underwent electroencephalography (EEG) and head magnetic resonance imaging (MRI) performed. The latter showed no pathological changes but the EEG record was pathognomonic for epilepsy, with the seizure activity registered in the fronto-temporal area of the patients' cerebrum (Fig. 2).

The patient had valproic acid introduced to the treatment. Due to the lack of correlation with appearance of seizures and methotrexate intake this drug was re-introduced. Despite the short abandonment of DMARD, JIA remained in remission and the girl showed no clinical or laboratory features of joint inflammation at that time.

During the year following the first seizure, due to the low JIA activity our 4-year-old girl was consulted by a rheumatologist in the outpatients clinic only. The patient's physician observed progressive deterioration of the patient clinical condition - her walk, previously normal for the age, began to be unstable, on a broadened base of her feet, which had no correlation with increased JIA activity. What is more, this previously cheerful and talkative girl appeared to have problems with pronouncing even the basic words, her contact with others began to be flat, and she had problems with following her mother's simplest instructions, which was not observed in the period before the seizures occurred. What has worried the rheumatologist was also observed by the consulting neurologist - despite valproic acid treatment seizure activity was still present, epileptic seizures with consciousness disturbances began to be an everyday problem for the patients family. The seizures were mainly myoclonic in type, neurological examination showed increased muscle tone and ataxia, confirmed by a positive Romberg test (Fig. 3). 
4-year old girl with noticeably higher frequency of epileptic seizures and worsening of neurological condition (for a few days: worse contact with the environment, no werbal contact possible, difficulties in feeding). During the examination: girl in a flat contact with surrounding environment, unable to follow simple commands, not answering questions. Tremor of upper limbs, numerous involountary movements within muscles of face and upper and lower limbs characteristics for choreoatetosis. Features of thoracic ataxia.

Fig. 3. Neurological consultation.
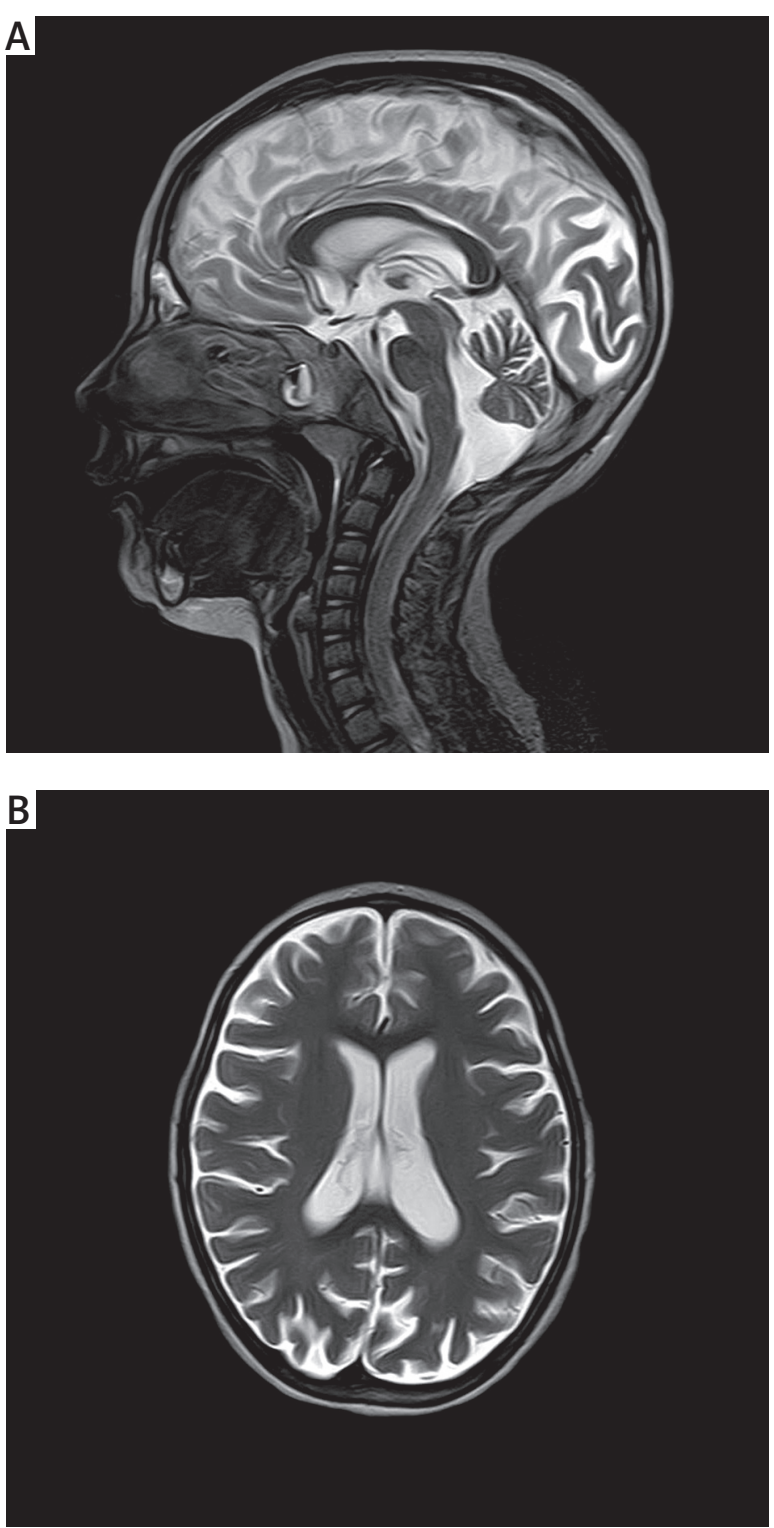

Fig. 4. MRI of patients brain with signs of cerebral and cerebellar atrophy.

Lamotrygine, levetiracetam and topiramate were gradually introduced to patient treatment with a little clinical effect. Due to rapid progression of the disease and noticeable deterioration of neurological condition of the patient, MRI of the brain was performed once again. In this MRI examination the picture was highly suggestive of the genetic disease being the cause of the appearance of these symptoms - hypoplasia of the cerebellum and widening of cerebral sulci were evident (Fig. 4).

The genetic tests confirmed our suspicions about the existence of the genetic disorders - a mutation in CLN2 gene was described, which is pathognomonic for one, inevitably fatal, disease from the group of lysosomal storage diseases - neuronal ceroid lipofuscinosis type II.

\section{Discussion}

Neuronal ceroid lipofuscinoses (NCLs) are among main causes of neurodegenerative changes observed in the paediatric population. Their incidence frequency varies between particular world regions, but diseases of this group are assessed to affect about $1: 100,000$ of live births in the European population [1].

Neuronal ceroid lipofuscinoses form a subgroup of lysosomal storage diseases, rare disorders causing accumulation of storage materials within the lysosome [2, 3]. These defects are inherited mainly in autosomal recessive pattern and are usually the effect of single lysosomal enzyme deficiency, leading to storage of cell metabolism waste products in neurons and other cells, progressively leading to deterioration of the patients neurological state, motor decline, epilepsy and loss of vision.

Neuronal ceroid lipofuscinoses are not a coherent group of diseases. They can be caused by a number of gene mutations [4], resulting in a wide array of disease onset age and variability of symptoms. Fourteen different genes involved in the pathogenesis of NCLs are recognised, but recently reports on new mutations causing disease onset have also been published [5, 6]. This high degree of genetic heterogeneity results in a diversity of the forms of the diseases - at this moment there are at least than ten types of NCLs described $[3,7]$. The most common ones are infantile neuronal ceroid lipofuscinosis (INCL), late infantile neuronal ceroid lipofuscinosis (LINCL) and juvenile neuronal ceroid lipofuscinosis [7, 8] (Table II). These diseases are caused by mutations in CLN1, CLN2 and CLN3 genes respectively.

Late infantile neuronal ceroid lipofuscinosis or neuronal ceroid lipofuscinosis type II was diagnosed in the case of the patient presented above. The course of the disease in our 4-year old girl was typical for LINCL, with no signs incipient neurodegenaration until reaching the age of 2. The first symptoms usually occurring in the course of LINCL were observed during routine examinations in our Clinic and comprised of hypotonia, gait disturbances, ataxia and developmental regression. Therapy-resistant epileptic seizures, typical in NCLs progression likewise, occurred as well. The key to the proper di- 
Table II. Forms of NCL

\begin{tabular}{|c|c|c|}
\hline \multicolumn{3}{|c|}{ Neuronal ceroid lipofuscinoses types and their clinical picture } \\
\hline NCL type & Clinical features & Gene involved \\
\hline 1 & $\begin{array}{l}\text { Infantile NCL. Age of onset: } 6 \text { months }-2 \text { years. } \\
\text { Symptoms: failure to thrive, microcephaly, myoclonic seizures }\end{array}$ & TPP-1/CLN1 \\
\hline 2 & $\begin{array}{l}\text { Late infantile NCL. Age of onset: } 2-4 \text { years. } \\
\text { Symptoms: ataxia, seizures, neurological deteriotarion, vision loss }\end{array}$ & TPP-1/CLN2 \\
\hline 3 & $\begin{array}{l}\text { Juvenile NCL. Age of onset: } 4-8 \text { years. } \\
\text { Symptoms: vision loss, seizures, ataxia }\end{array}$ & CLN3 \\
\hline 4 & $\begin{array}{l}\text { Adult NCL. Age of onset: before } 40 \text {. } \\
\text { Symptoms: mild deterioration of neurological condition, no problems with vision }\end{array}$ & CLN6, DNAJC5 \\
\hline 5 & $\begin{array}{l}\text { Finnish Late Infantile NCL. Age of onset: } 4-7 \text { years. } \\
\text { Symptoms: motor decline, visual loss, seizures }\end{array}$ & CLN5 \\
\hline 6 & Variant Late Infantile. Age of onset: 4-7 years. Symptoms: as above & CLN6 \\
\hline 7 & Variant Late Infantile. Symptoms as in LINCL but more severe course regarding seizures & MFSD8 \\
\hline 8 & CLN8 Northern Epilepsy - the mildest NCL manifesting in epileptic seizures & CLN8 \\
\hline 9 & Mutation described in a few German patients & unknown \\
\hline 10 & $\begin{array}{c}\text { CLN10, Cathepsin D deficiency. Symptoms: seizures before birth and in neonatal period. } \\
\text { Usually fatal in first days of life }\end{array}$ & CTSD \\
\hline
\end{tabular}

agnosis of our patient was the finding of cerebral and cerebellar atrophy in MRI performed as a result of the patient rapid progression suggested for neurodegenerative changes. The only difference between classical course of LINCL and that one observed in our patient is lack of visual impairment at this moment, but as studies show, the diagnosis of visual loss may be delayed due to the existence of neurological defects of the patient resulting in poor compliance during routine ophthalmologic examinations. Death is inevitable inscribed in the course of the disease and usually happens at the age of 10-15 years.

The molecular background behind the LINCL onset is a mutation of CLN2, a gene responsible for the proper function of tripeptidyl peptidase 1 (TPP-1), a lysosomal protease which is associated with the failure to degrade specific neuropeptides and a subunit of ATP synthase [9]. According to the University College London database there are over 100 identified mutations in TPP-1 which may lead to LINCL onset. The most common ones are Arg208Stop and a c.509-1G > C [5]. Judging by the rapid development of molecular biology techniques one may think that causal treatment of NCLs is within reach. Unfortunately, the future may not be so bright: there are many studies on the potential role of gene and enzyme replacement therapy in progress currently, but there are many obstacles on their way. The main one is creating a viral vector capable of transmitting DNA into the affected tissue and this is far more challenging than in other genetic diseases, e.g. lipoprotein lipase deficiency as the potential vector must be able to cross the bloodbrain barrier [7].
TPP-1 may not only be a promising target for further research in the field of neurology. Some recent studies show a potential role of this particle in the pathogenesis of rheumatoid arthritis, as TPP-1 altered expression in fibroblast-like synovial cells was associated with development of this rheumatic disease [10].

\section{Summary}

Taking into consideration the result of this study it is tempting to suppose that JIA and LINCL development in one patient was not just a pure coincidence. Nevertheless, in the literature there are no reports of TPP-1's role in the pathogenesis of IIA and this subject needs further research to be conducted.

The authors declare no conflict of interest.

\section{References}

1. Claussen M, Heim P, Knispel J, et al. Incidence of neuronal ceroid-lipofuscinoses in West Germany: variation of a method for studying autosomal recessive disorders. Am J Med Genet 1992; 42: 536-538.

2. Meikle PJ, Hopwood JJ, Clague AE, Carey WF. Prevalence of lysosomal storage disorders. JAMA 1999; 281: 249-254.

3. Schulz $A$, Kohlschütter $A$. NCL Disorders: frequent causes of childhood dementia. Iran J Child Neurol 2013; 7: 1-8.

4. Williams RE, Mole SE. New nomenclature and classification scheme for the neuronal ceroid lipofuscinoses. Neurology 2012; 79: 183-191

5. Canafoglia L, Morbin M, Scaioli V, et al. Recurrent generalized seizures, visual loss and palinopsia as phenotypic features of 
neuronal ceroid lipofuscinosis due to progranulin gene mutation. Epilepsia 2014; 55: 56-59.

6. Yu F, Xiao-Min L, Yin-He Ch, et al. A novel CLN2/TPP1 mutation in a patient with late infantile neuronal ceroid lipofuscinosis. Neurolog Sci 2015; 36: 1917-1919.

7. Geraets R, Koh S, Hastings M, et al. Moving towards effective therapeutic strategies for neuronal ceroid lipofuscinosis. Orphanet J Rare Dis 2016; 11: 40.

8. Jadav R, Sinha S, Yasha TC. Clinical, electrophysiological, imaging, and ultrastructural description in 68 patients with neuronal ceroid lipofuscinoses and its subtypes. Pediatric Neurology 2014; 50: 85-95.

9. Chang-Gong L, Sleat D, Donelly R, et al. Structural organization and sequence of CLN2, the defective gene in classical late infantile neuronal ceroid lipofuscinosis. Genomics 1998; 50: 206-212.

10. Qing YF, Zhou JG, Zhao MC, et al. Altered expression of TPP1 in fibroblast-like synovial cells might be involved in the pathogenesis of rheumatoid arthritis. Rheumatol Int 2012; 32: 2503 2510 . 